\title{
Optimization of the laser beam propagation conditions in the prism stretcher of THL-100 multiterawatt laser system
}

\author{
M.V. Ivanov ${ }^{\text {a }}$, S.V. Alekseev ${ }^{\mathrm{a}}$, N.G. Ivanov ${ }^{\mathrm{a}}$, V.F. Losev ${ }^{\mathrm{a}, \mathrm{b}}$ \\ ${ }^{a}$ Institute of high current electronics Siberian Branch RAS, Akademichesky Avenue 2/3, 634055, \\ Tomsk, Russia, mikhan.iv@gmail.com; ${ }^{b}$ National Research Tomsk Polytechnic University, Lenin \\ Avenue 30, 634050, Tomsk, Russia
}

\begin{abstract}
The influence of the optical inhomogeneities effect of the prism stretcher elements on the spatial, angular and spectral parameters of the transmitted radiation and the degree of change in the phase aberrations of the laser beam during its propagation in the stretcher are studied. It is shown that the prism material does not allow transmitting of a required diameter of $75 \mathrm{~mm}$ without linear distortions of the beam. The maximum intensity and beam diameter allowing to amplify the picosecond pulses in $\mathrm{XeF}(\mathrm{C}-\mathrm{A})$ amplifier are determined.
\end{abstract}

Keywords: Prism stretcher, femtosecond laser pulse, linear distortion, beam divergence, inhomogeneity.

\section{INTRODUCTION}

In the last decade the research and developments aimed at creating of the laser systems of terawatt and petawatt power level is rapidly developed [1-4]. The great interest in this area of research is due to the fact that the achievement in laser pulse of ultra-high power opens an opportunities for research in the rapidly developing fields of modern physics. All high power installations work on the solid-state active media which emit in the infrared spectrum region $(0.8-1 \mathrm{~m} \mu)$.

In the Institute of High Current Electronics SB RAS (Tomsk) on the initiative and support of academician G.A. Mesyats the THL-100 hybrid laser system operating in the visible spectrum range $(475 \mathrm{~nm})$ was created [5-11]. The accumulated experience of the high power gas lasers development was used for it creation [12-14].Visible range gives the new opportunities for high power laser beams interaction with matter. Obtaining such a powerful radiation in the visible range by the second harmonic generation in high-power infrared laser systems is limited by the technical capabilities of non-linear crystals manufacturing. The maximum output power obtained by this method at a $400 \mathrm{~nm}$ wavelength is $4 \mathrm{TW}$ [15]. In the laser system of THL-100 at the first research stage the power level of 14 TW was reached [9-11] and the achievement of 50-100 TW range is planned.

The laser system of THL-100 consists of a Ti:sapphire start complex, prism stretcher, photochemical XeF(C-A) amplifier and glass compressor. Start complex emits the $50 \mathrm{fs}$ pulses with energy at the second harmonic (475 $\mathrm{nm}$ ) up to $20 \mathrm{~mJ}$. Prism stretcher due to the negative dispersion increases the pulse duration from a femtosecond to the 1-3 ps range. This chirped pulse is amplified in the active medium of $\mathrm{XeF}(\mathrm{C}-\mathrm{A})$ amplifier with an aperture of $24 \mathrm{~cm}$. Active medium is created in $\mathrm{XeF}_{2} / \mathrm{N}_{2}$ mixture by VUV radiation xenon which is excited by a high-power electron beam. In order to achieve the gain saturation of the amplified signal the multipass optical scheme (33 passes) is used. After amplification the laser pulse is compressed by the glass compressor to initial duration of $50 \mathrm{fs}$. Stretching of the transform-limited pulse up to picosecond duration is necessary for its more efficiency amplification in the active medium of $\mathrm{XeF}(\mathrm{C}-\mathrm{A})$ amplifier and reducing of the radiation exposure on its optical components. It is very important that the input laser beam of $\mathrm{XeF}(\mathrm{C}-\mathrm{A})$ amplifier has a good uniformity in intensity and spectral compositions. However in case the propagation of ultrashort laser pulse in prism stretcher both linear and non-linear distortion of the laser beam in the prisms can occur. In addition for full compensation of angular dispersion of the output beam a very accurate alignment of the prisms in three dimensions have to be provided. Therefore the very strict requirements are lodged to the prism stretcher which are necessary to provide for its efficient operation.

To determine the optimal operating conditions of the prism stretcher we carried out the experimental and theoretical studies of passage through it of the femtosecond radiation pulse at a $475 \mathrm{~nm}$ wavelength with varying intensity and diameter of the laser beam. Herewith the spatial, angular and spectral parameters of the transmitted radiation were recorded. 


\section{THE EQUIPMENT AND TECHNIQUES}

In the experiments the radiation from a solid-state start complex was passed through a prism stretcher. The laser beam energy was varied in the range of $0.1-1 \mathrm{~mJ}$. The optical stretcher scheme allows providing a negative dispersion of the laser beam with a diameter of 10 to $75 \mathrm{~mm}$. The output beam from the stretcher was went out at a slight angle to input beam in the vertical direction. To determine the necessary alignment accuracy a computer model of a prism stretcher was modeled in Zemax program. It takes into account only the geometric distortion of the beam. Experimental and theoretical simulation is performed in two stretcher configurations. In the first case after passing through the prisms the beam is back reflected by a mirror at a slight angle $(\sim 0.5$ degrees $)$ to the vertical direction to the incident radiation to take it out of the stretcher, Figure 1.

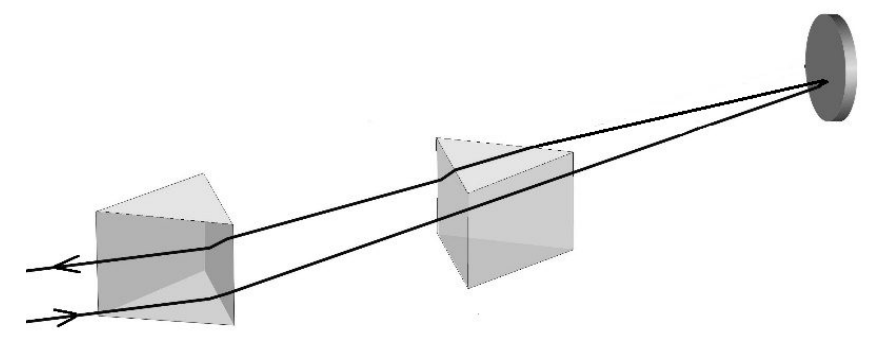

Figure 1. Simulated stretcher scheme. The reverse radiation passes through the stretcher at an angle to the vertical line.

In the second case the beam was mounted by two mirrors and it was returned strictly parallel to input beam, Figure 2 . The calculation for a beam diameter of $45 \mathrm{~mm}$ at three wavelengths of $465,475,485 \mathrm{~nm}$ was performed. These wavelengths correspond to the central and boundary values of our beam.

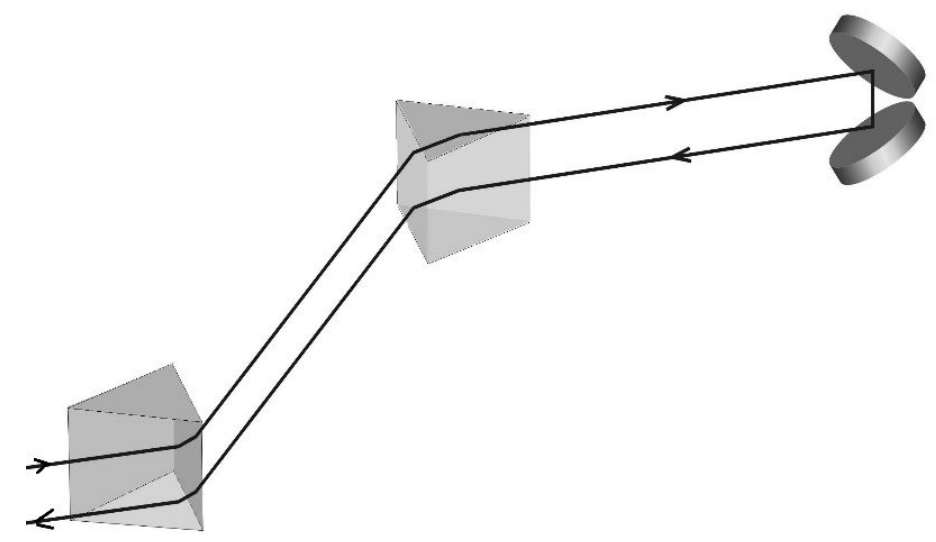

Figure 2. Simulated stretcher scheme with roof-mirror.

In our experiments it was found that even with a small misalignment of the optical stretcher scheme the full compensation of angular dispersion in the output beam is not implemented. In this regard, the calculations were carried out to determine the accuracy of the installation angles of the prisms in three dimensions. Control these angles magnitude was determined by the beam size in the far field, so that it does not exceed the Airy disk. In these configurations the 
stretcher provides the maximum possible stretching of the pulse duration. After the passage through the stretcher the duration of the chirped pulse becomes $2.6 \mathrm{ps}$. The distance between the prisms is equal $9.6 \mathrm{~m}$.

After careful adjustments of the stretcher, allocation of spectrum in the cross section of the beam was experimentally measured. To reduce the intensity of the radiation on prisms the laser beam is expanded by the mirror telescope with a magnification $\mathrm{M}=3$. The size of the beam in the prisms was $45 \mathrm{~mm}$. The spectrum distribution across the beam experimentally was measured in the two schemes. The first case is equivalent to a simulated, shown in Figure 1. In the second case, the corner reflector was replaced by a flat mirror, so that the beam in stretcher was returned exactly the same way as the direct passage. In such scheme a beam-splitter, which fully reflects infrared radiation and transmits visible spectral range, was set in front of the spectrometer for measuring the spectrum. To find the dimensions of the beam, in which the angular dispersion is fully compensated in the stretcher, the initial beam was limited by diaphragm, which was set before KDP. The Avantes spectrometer was used for control over the angular dispersion of the output beam registering the spectral composition of the output beam. It was located 30 meters from the beam-splitter. At the same time the monitoring beam with a diameter of $15 \mathrm{~mm}$ was recorded in three areas - central, left and right each with a diameter of $6 \mathrm{~mm}$. The influence of the stretcher on the divergence of the laser emission was experimentally measured using the narrow-band diode laser radiation at a wavelength of $488 \mathrm{~nm}$. Beam divergence measured by the next method: the beam diameter in the prisms was $50 \mathrm{~mm}$, the beam is directed onto a spherical mirror with a focal length of 11 meters, in which the focal waist located turret aperture with a diameter from 0.5 to $5 \mathrm{~mm}$, with increments of $0.5 \mathrm{~mm}$. Ophir Nova II calorimeter was set up after the diaphragm.

\section{RESULTS}

Through computer modeling of the prism stretcher were defined limit values of misalignment angles of prism that the output beam in the aberration-free focusing lens does not exceed the size of the Airy disk. Figure 3 shows a spot diagram of radiation in the far field in the simulation scheme with corner reflector. On the left diagram, the deviation from Brewster's angle of the prisms is 0.4 degrees. With this amount of deflection angle of rotation of the prism beam size at the focus is less than the Airy disk. However, the wavelengths of 465 and 485 are located on the border of the Airy disk vertically
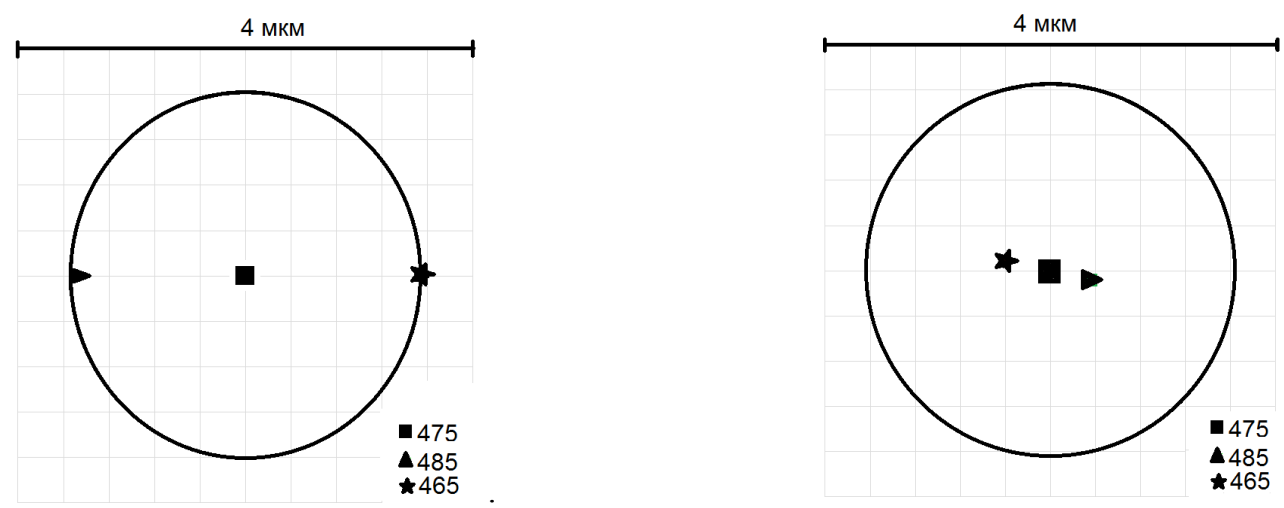

Figure 3. Spot diagram at alignment deviations (left - Brewster angle, right - vertical angles).

The right diagram of the figure 3 shows the effect of the deflection angle from the vertical axis of prism in the simulation scheme with corner reflector. In this case, the deviation angle is a 5 degree. This is the maximum possible angle of prisms deviation from vertical axis. It is seen that the boundary wavelengths of our beam are separated by a short distance within the Airy disk. Thus, we can conclude that the alignment in the plane of the stretcher in this scheme is not as critical as the setting angle of Brewster prisms. Figure 4 (left) is a spot diagram of the far field radiation in the simulation scheme of the stretcher with the reverse passage of radiation at an angle to the vertical axis. In this case, the deviation of the prisms from Brewster's angle equals the 0.34 degrees. Position accuracy in this case is slightly higher than in the previous case. 

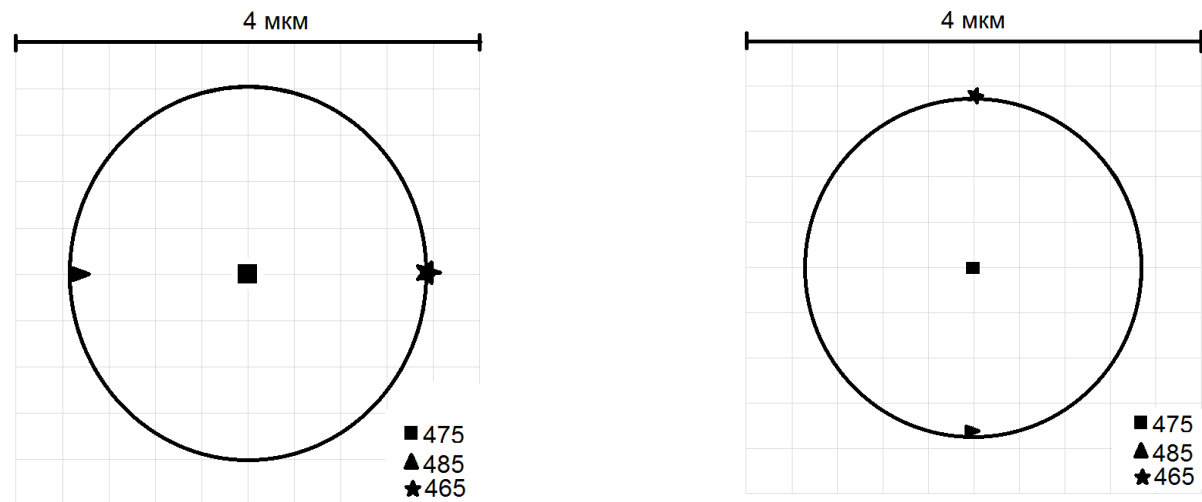

Figure 4. Spot diagram at alignment deviations (left - Brewster angle, right - vertical angles).

However, the accuracy setting the prisms from vertical axis is significantly higher and it is 1.2 degrees. In this case, the beam dispersion is stretched in a vertical plane, which is shown in Figure 4 (right).

Figure 5 shows the experimental dependence of the spectral distribution of the beam. From this graph can see that the spectrum of the beam is not evenly distributed across the beam. The laser beam before the stretcher has a maximum at $475 \mathrm{~nm}$ and the spectral width of $\sim 6 \mathrm{~nm}$ at half maximum. After passing through the stretcher in the central part of the beam only wavelengths $473-475 \mathrm{~nm}$ remain. The spectrum with shorter wavelengths is at the right part of the beam and the spectrum with longer wavelengths is at the left part of the beam. A similar situation was observed in the vertical plane. Further work with the beam in the laser system THL-100 is not possible, as part of the spectrum, located on the periphery of the beam will be lost in the spatial filter and the subsequent optical elements that lead to a narrowing of the spectrum up to $2.5 \mathrm{~nm}$. It corresponds to the duration of $130 \mathrm{fs}$ for transform-limited pulse. Based on computer simulations it can conclude that this spectrum distribution of the beam after the stretcher can be observed at non-optimal installation angles of the prisms. However, the accuracy of the stretcher alignment was higher than calculated angles in the computer model. Most likely the heterogeneity of the prism material leads to the impossibility of full compensation of angular dispersion while operating with diameters greater than $45 \mathrm{~mm}$.

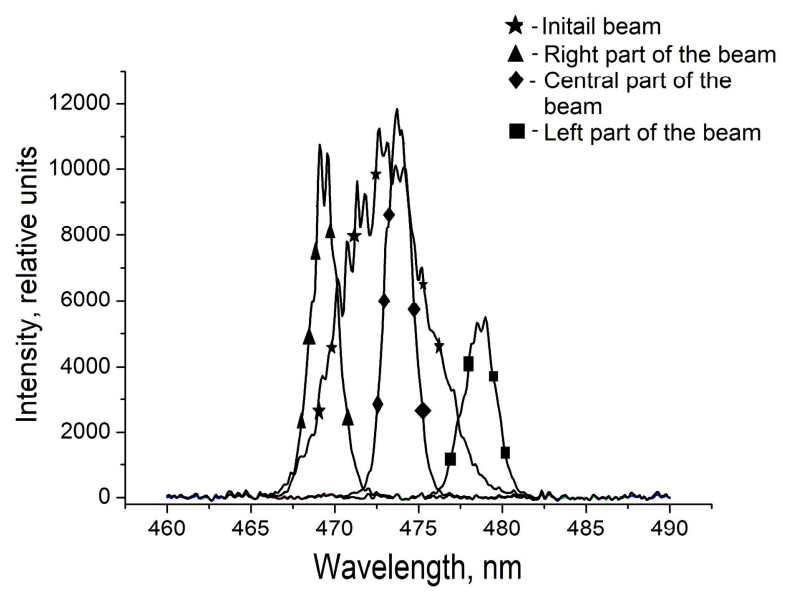

Figure 5. Spectrum distribution of the beam after the stretcher.

To exclude the influence of the prisms tilt angles the modeling experiments were conducted when the beam was directed straight back through the stretcher. Wherein further to reduce the influence of glass inhomogeneity the beam diameter at the first harmonic was limited to $2 \mathrm{~mm}$. In this case, the diameter of the beam passing through the prism was $10 \mathrm{~mm}$ and its an energy was $20 \mu \mathrm{J}$. In consequence of the passage of such a scheme the beam is turned in polarization rotator in 90 degrees, and thus the experimental dependence of the spectrum corresponds to the horizontal plane of the simulated stretcher. The distribution of the emission spectrum across the beam in such a stretcher configuration is illustrated in Figure 6. In this case, the emission spectrum in the central part of the beam coincides with the initial spectrum width. At the periphery of the beam still observed the less spectrum narrowing than in the case shown in Figure 5. 


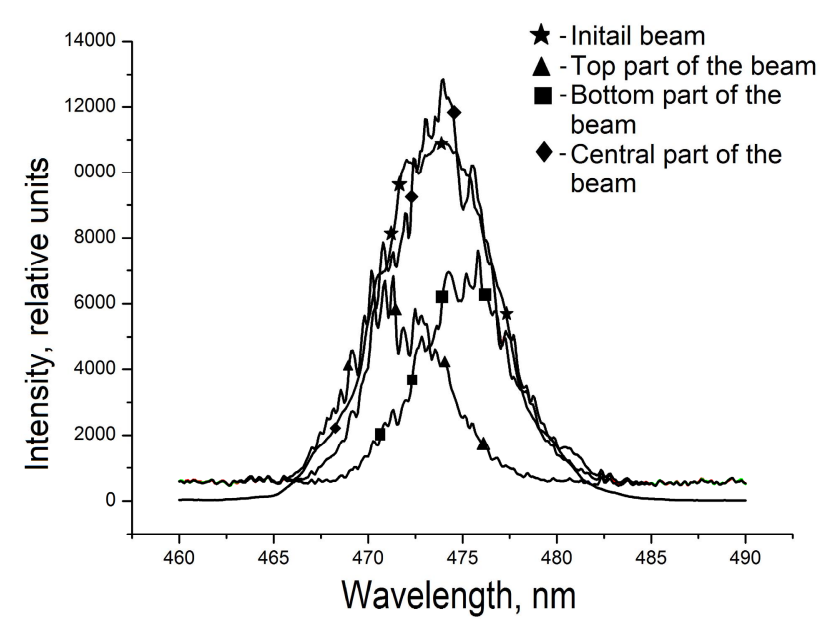

Figure 6. Spectrum distribution of the beam after the stretcher.

Figure 7 shows the energy diagram of the beam in the far field after passing through the stretcher at a wavelength of 488 $\mathrm{nm}$. This figure shows that $84.6 \%$ of the beam energy is located at 2.3 diffraction angles. It can be concluded that the divergence of the beam deteriorates in consequence of the poor quality of the prism material also as non-axial output of radiation from the telescope. The improvement of divergence in the stretcher can be reached by additional aberrations, such as the installation of a plane-parallel plate at some angle inside of the stretcher or installation of prisms not parallel to each other. In addition, divergence may be improved by using a spatial filter however it will lead to a narrowing of the spectrum.

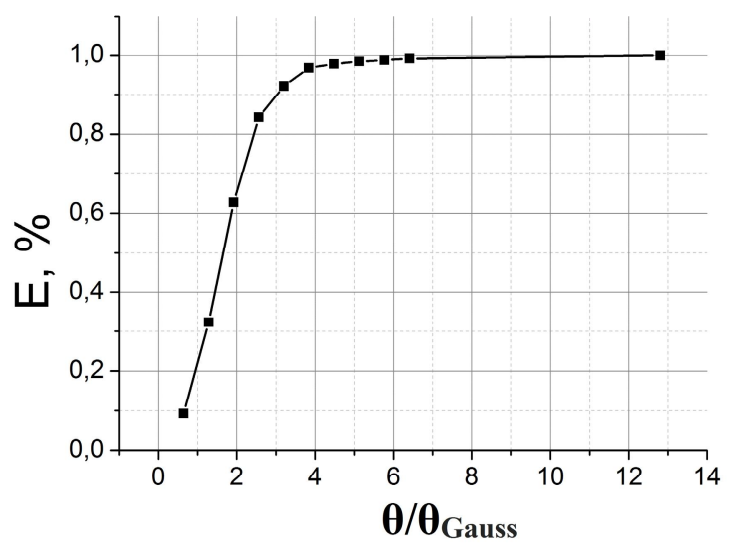

Figure 7. The divergence of radiation at a wavelength of $488 \mathrm{~nm}$ after stretcher passing.

Thus by computer modeling in program Zemax the alignment prisms stretcher angles was identified at which the output beam has no significant distortion. Based on calculated accuracy of alignment the uncompensated angular dispersion in the stretcher should be not observed. However even with a low energy eliminating the nonlinear optical effects in stretcher cannot avoid uncompensated angular dispersion experimentally. This is due to the heterogeneity of the prism material. Full compensation of the stretcher angular dispersion can be achieved by introducing additional aberrations in the stretcher, for example, this can be achieved by setting the prisms having non optimal angles. It is easy to implement for the narrow-band radiation of a diode laser at a wavelength of $488 \mathrm{~nm}$, however, it impossibly to realize for broadband femtosecond laser radiation. As a result of this work it was shown that in the stretcher of femtosecond laser system THL100 there are serious problems with laser beams diameter greater than $1 \mathrm{~cm}$. 


\section{ACKNOWLEDGMENT}

This work has been supported by "Russian scientific Foundation" project \# 15-19-10021

\section{REFERENCES}

[1] Colin Danson, David Hillier, Nicholas Hopps, and David Neely. "Petawatt class lasers worldwide," High Power Laser Science and Engineering 3, 14 (2015).

[2] E.I. Moses, "Ignition on the National Ignition Facility: a path towards inertial fusion energy," Nuc. Fus., 49, 104022 (2009).

[3] Xiaoming Lu, Xiaoyan Liang, Yuxin Leng et al. "Improvement of the large aperture Ti:sapphire amplif iers in the petawatt femtosecond laser system at SIOM.," CHINESE OPTICS LETTERS 8, 1008 (2010).

[4] A.J. Bayramian et. al., "The Mercury Project: A High Average Power, Gas-Cooled Laser For Inertial Fusion Energy Development," Fus. Sci. \& Tech. 52, 383-387 (2007).

[5] Losev, V., Alekseev, S., Ivanov, N., Kovalchuk, B., Mikheev, L., Mesyats, G., Panchenko, Yu., Ratakhin, N. \& Yastremsky, A. "Development of a hybrid (solid state/gas) femtosecond laser system of multiterawatt peak power," Proc. SPIE.7751, 7751(2010).

[6] Losev, V., Alekseev, S., Ivanov, N., Kovalchuk, B., Mikheev, L., Mesyats, G., Panchenko, Yu., Puchikin, A., Ratakhin, N. \& Yastremsky, A. "Development of a 100-terawatt hybrid femtosecond laser system," Proc. SPIE. 7993, 421-425. (2011).

[7] Alexeev, S.V., Ivanov, N. G., Kovalchuk, B.M., Losev V.F., Mesyats, G.A., Mikheev, L.D., Panchenko, Yu.N., Ratakhin, N.A., and Yastremsky, A.G. "Hybrid femtosecond laser system THL-100 on the base of XeF(C-A) amplifier," Atmospheric and Oceanic Optics 25, 221-225 (2012).

[8] Losev V.F., Alekseev S.V., Aristov A.I., Ivanov N.G., Kovalchuk B.M, Mesyats G.A., Mikheev L.D., Panchenko Yu.N., and Ratakhin N.A. "Hybrid Multi-Terawatt Laser System of Visible Spectral Range," Proc. SPIE. 8677, 8677 (2012).

[9] S.V. Alekseev, A.I. Aristov, N.G. Ivanov, B.M. Kovalchuk, V.F. Losev, G.A. Mesyats, L.D. Mikheev, Yu.N. Panchenko, and N.A. Ratakhin "Multiterawatt Femtosecond Laser System In the Visible with Photochemically Driven Xef(C-A) Boosting Amplifier," Laser and Particle Beams. 31, 17-21 (2013).

[10] S.V. Alekseev, A.I. Aristov, N.G. Ivanov, B.N. Koval'chuk, V.F. Losev, G.A. Mesyats, L.D. Mikheev, Yu.N. Panchenko, N.A. Ratakhin, "Multiterawatt femtosecond hybrid system based on a photodissociation XeF(C-A) amplifier in the visible range," QUANTUM ELECTRON 42, 377-378 (2012).

[11] S. V. Alekseev, A. I. Aristov, Ya. V. Grudtsyn, N. G. Ivanov, B. M. Koval'chuk, V. F. Losev, S. B. Mamaev, G. A. Mesyatsb, L. D. Mikheev, Yu. N. Panchenkoa, A. V. Polivinb, S. G. Stepanovb, N. A. Ratakhinca, V. I. Yalovoi, A. G. Yastremskii "Visible-range hybrid femtosecond systems based on a $\mathrm{XeF}(\mathrm{C}-\mathrm{A})$ amplifier: state of the art and prospects," Kvant. electron. 43, 190-200 (2013).

[12] N. G. Ivanov, V. F. Losev, Ė. I. Naats, V. V. Ryzhov, I. Yu. Turchanovskii, A. G. Yastremskii "XeCl laser with an output energy 200," Kvant. electron. 24, 688-690. (1997).

[13] Y.I. Bychkov, N.G. Ivanov, I. N. Konovalov, et. "A XeCl Laser-excited by a microsecond electron-beam," Kvant. electron.10, 1510-1512. (1983).

[14] Y.I. Bychkov, N.G. Ivanov, V. F. Losev, G.A. Mesyats "XeCl-laser with energy generation 150 J," Pis'ma v JTPh 14, 566-569 (1988).

[15] Ozaki, T., Keiffer, J.-C., Toth, R., Fourmaux, S., Bandulet, H., "Experimental prospects at the Canadian advanced laser light source facility," Laser and Particle Beams 24, 101-106 (2006). 